\title{
The New Science of Complexity
}

\author{
JOSEPH L. MCCAULEY* \\ Physics Department, University of Houston, Houston, TX 77204, USA
}

(Received 9 October 1996)

\begin{abstract}
Deterministic chaos, and even maximum computational complexity, have been discovered within Newtonian dynamics. Economists assume that prices and price changes can also obey abstract mathematical laws of motion. Sociologists and other postmodernists advertise that physics and chemistry have outgrown their former limitations, that chaos and complexity provide new holistic paradigms for science, and that the boundaries between the hard and the soft sciences, once impenetrable, have disappeared like the Berlin Wall. Three hundred years after the deaths of Galileo, Descartes, and Kepler, and the birth of Newton, reductionism appears to be on the decline, with holistic approaches to science on the upswing. We therefore examine the evidence that dynamical laws of motion may be discovered from empirical studies of chaotic or complex phenomena, and also review the foundation of reductionism in invariance principles.
\end{abstract}

Keywords: Complexity, Simulations, Laws of nature, Invariance principles, Socio-economic sciences

\section{SOCIO-ECONOMIC FIELDS AND "SYSTEM THEORY"}

I define "system theory" broadly to include mathematical models written in terms of systems of deterministic and stochastic ordinary and partial differential equations, iterated maps, and deterministic and stochastic automata.

By a mathematical law of nature, in contrast to the models and simulations of "system theory", I mean an empirically verified law of motion, a correct mathematical law of time-evolution.
Galileo and Kepler discovered the simplest special cases. Their local laws were generalized by Newton to become three universally valid (global) laws of motion, along with a universal law of gravity. Newton's laws are "universal" in the following sense: they can be verified, often with very high decimal precision, regardless of where and when on earth (or on the moon or in an artificial satellite) careful, controlled experiments, or careful observations, are performed.

"Laws" of economics, "laws" of human behavior, and the Darwin-Wallace "laws" of fitness,

*Tel.: 713-743-3528. Fax: 713-743-3589. E-mail: kaos@george.phys.uh.edu. 
competition, selection and adaptation are sometimes mentioned in the same context as laws of motion of inanimate matter (physics and chemistry), although since the time of Galileo the word "law" in the first three cases does not have the same import as in the case of physico-chemical phenomena. Confusion over what constitutes a law of nature is ancient: Aristotle invented a purely qualitative, holistic approach to the description of nature. Not recognizing any distinction between the different uses of the idea of natural law, he lumped together as "motion" the rolling of a ball, the education of a boy, and the growth of an acorn [1].

In attempts to describe socio-economic phenomena from the standpoint of system theory it is Platonically assumed that the probability distributions describing prices and price changes, or other social factors, are determined by an objective mathematical law that governs how the economic system evolves [2]. This assumption is not only sufficient but is also necessary if the idea of mathematical law in economics is to make sense.

In the first chapter of his text on elementary economics [3], Samuelson tries to convince both the reader and himself that the difference between the socio-economic fields and the laws of physics is blurry, that economics can be treated as if it would also be a science subject to mathematical law. Samuelson claims that physics is not necessarily as lawful as it appears, that the laws of physics depend subjectively on one's point of view. His argument is based on a nonscientific example of ambiguity from the visual perception of art (Fig. 1) and is genetically related through academic mutation and evolution to a viewpoint that has been advanced by the postmodernist and deconstructionist movement in art, literature, philosophy, psychology, and sociology. The latter argue that a text has no more meaning than the symbols on a printed page, that there is no universal truth, and therefore no universal laws of nature, and that Platonic-Ptolemeic astronomy and Aristotelian physics are still just as valid as fields of scientific study as are physics and as-

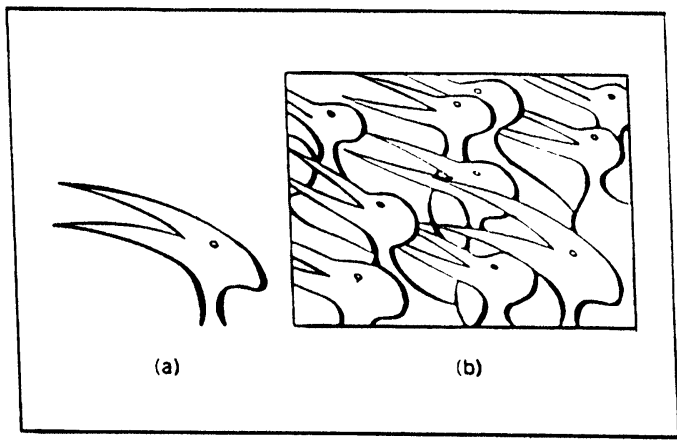

FIGURE 1 Samuelson's question: Is it a bird or an antelope? Answer: neither, it's a continuous line between two points plus a closed curve that, unlike both birds and antelopes, is topologically equivalent to a straight line plus a circle (from Samuelson [3]).

tronomy since Galileo and Kepler (who revived the spirit of Archimedes).

Samuelson notes that physics relies on controlled experiments, and adds that in the socioeconomic fields it is generally impossible to perform controlled experiments. This is not an excuse for bad science: controlled experiments are also impossible in astronomy where mathematical laws of nature have been verified with high decimal precision. See also Feynman [4] for criticism of the lack of isolation of cause and effect in the psycho-social fields.

Platonists in mathematics [5] believe that mathematical laws exist objectively and govern everything that happens. Physics is neither Aristotelian (qualitative and "holistic") nor Platonic (relying upon wishful thinking, because the presumed mathematical laws are not grounded in careful, repeatable empiricism).

The divorce of the study of nature from Platonic and Aristotelian notions was initiated by Galileo and Descartes [6], but that divorce was not complete: with Galileo's empirical discoveries of two local laws of nature physics became a precise mathematico-empirical science. Biology, excepting the study of heredity since Mendel and excepting biochemistry and biophysics since the discovery of quantum mechanics, has continued through the age of Darwin and beyond as a 
largely descriptive science in the tradition of Aristotle, with reliance on vague (mathematically undefined) sociological notions like "competition, natural selection and adaptation".

I will argue that economic and other social phenomena lie beyond the bounds of understanding from the standpoint of dynamical modeling that attempts to describe the time-evolution of systems, even if the goal is merely to extract the crudest features like coarsegrained statistics. In order to make my argument precise, I first review the necessary background on deterministic dynamical systems.

\section{WHAT DOES “NONINTEGRABLE” MEAN?}

The ambiguity inherent in early attempts to distinguish "integrability" from "nonintegrability" was expressed poetically by Poincaré, who stated that a dynamical system is generally neither integrable nor nonintegrable, but is more or less integrable [7]. Modern mathematicians have managed to give some precise definitions of integrability [8] that are hard to translate into simpler mathematics. Below, I describe what "nonintegrability" means analytically and, in part, geometrically.

For the sake of precision I frame my discussion in the context of flows in phase space,

$$
\frac{\mathrm{d} x}{\mathrm{~d} t}=V(x),
$$

where phase space is a flat inner product space so that the $n$ axes labeled by $\left(x_{1}, \ldots, x_{n}\right)$ can be regarded as Cartesian [9], and $V(x)$ is an $n$-component time-independent velocity field. Newtonian dynamical systems can always be rewritten in this form whether or not the variables $x_{i}$ defining the system in "physical" three-dimensional space are Cartesian). Flows that preserve the Cartesian volume element $\mathrm{d} \Omega=\mathrm{d} x_{1}, \ldots, \mathrm{d} x_{n}$ are defined by $\nabla \cdot V=0$ (conservative flows) while driven dissipative-flows correspond to $\nabla \cdot V \neq 0$, where $\nabla$ denotes the Cartesian gradient in $n$ dimensions. For a velocity field whose components satisfy the condition $V_{1}+\cdots+V_{n}=0$, then the global conservation law $x_{1}+\cdots+x_{n}=C$ follows. This abstract case includes chemically reacting systems with concentration $x_{i}$ for species $i$.

For a flow and for any initial condition $x_{0}$ the solution $x_{i}(t)=U(t) x_{i 0}$ has no finite time singularities [10] because singularities of trajectories of flows are confined to the complex time plane: the time evolution operator $U(t)$ exists for all real finite times $t$ and defines a one-parameter transformation group with inverse $U^{-1}(t)=U(-t)$, so that one can in principle integrate backward in time, $x_{0 i}=U(-t) x_{i}(t)$, as well as forward. Drivendissipative flows are therefore time-reversible (a diffusion approximation breaks time reversibility).

The Lorenz model

$$
\begin{aligned}
\frac{\mathrm{d} x_{1}}{\mathrm{~d} t} & =\sigma\left(x_{2}-x_{1}\right), \\
\frac{\mathrm{d} x_{2}}{\mathrm{~d} t} & =\rho x_{1}-x_{2}-x_{1} x_{3}, \\
\frac{\mathrm{d} x_{3}}{\mathrm{~d} t} & =-\beta x_{3}+x_{1} x_{2}
\end{aligned}
$$

defines a phase flow and is therefore perfectly time-reversible, even in the chaotic regime. This model may describe a chemically reacting system if $\beta=0$ and $\sigma=\rho=1$, in which case the flow is driven-dissipative but is not chaotic.

"Nonintegrable" does not mean not solvable: any flow, even a critical, chaotic or complex one, has a unique, well-defined solution if the velocity field $V(x)$ is at least once continuously differentiable with respect to all of the $n$ variables $x_{i}$. If, in addition, the velocity field is analytic in those variables then the power series

$$
x_{i}(t)=x_{i 0}+t\left(L x_{i}\right)_{0}+t^{2}\left(L^{2} x_{i}\right)_{0} / 2+\cdots,
$$

where $L=V \cdot \nabla$, has a nonvanishing radius of convergence, so that the solution of (1) can in 
principle be described by the power series (2) combined with analytic continuation for all finite times [11]. Both deterministic chaotic and complex flows are precisely determined over any desired number of finite time intervals by analytic formulae. The Lorenz model (1b) provides a chaotic example.

A completely integrable ("integrable") dynamical system has $n-1$ global time-independent first integrals (conservation laws) $G_{i}\left(x_{1}, \ldots, x_{n}\right)$ $=C_{i}$ satisfying the linear partial differential equation

$$
\frac{\mathrm{d} G_{i}}{\mathrm{~d} t}=V \cdot \nabla G_{i}=V_{k} \frac{\partial G_{i}}{\partial x_{k}}=0
$$

along any streamline of the flow. The global flow is then a time-translation for all finite times $t$ in the Lie coordinate system

$$
\begin{gathered}
y_{i}=G_{i}\left(x_{1}, \ldots, x_{n}\right)=C_{i}, \quad i=1, \ldots, n-1, \\
y_{n}=F\left(x_{1}, \ldots, x_{n}\right)=t+D,
\end{gathered}
$$

and the flow is confined to a two-dimensional manifold that may be either flat or curved, and is determined by the intersection of the $n-1$ global conservation laws [9]. In the description (4) of the flow all effects of interactions have been eliminated globally via a coordinate transformation. The transformation (4) "parallelizes" (or "rectifies" [10]) the flow: the streamlines of (1) in the $y$-coordinate system are parallel to a single axis $y_{n}$ for all times, where the time evolution operator is a uniform time-translation $U(t)=$ $\mathrm{e}^{t d / d y_{n}}$. Eisenhart asserted formally, without proof, that all systems of differential equations (1) are described by a single time translation operator [12], but this is possible globally (meaning for all finite times) only in the completely integrable case.

Algebraic or at least analytic conservation laws [13] have generally been assumed to be necessary in order to obtain complete integrability. For example, Euler's description of a torque-free rigid body [9]

$$
\begin{aligned}
\frac{\mathrm{d} L_{1}}{\mathrm{~d} t} & =a L_{2} L_{3}, \\
\frac{\mathrm{d} L_{2}}{\mathrm{~d} t} & =-b L_{1} L_{3}, \\
\frac{\mathrm{d} L}{\mathrm{~d} t} & =c L_{1} L_{2}
\end{aligned}
$$

with positive constants $a, b$, and $c$ satisfying $a-b+c=0$, defines a phase flow in three dimensions that is confined to a two-dimensional sphere that follows from angular momentum conservation $L_{1}^{2}+L_{2}^{2}+L_{3}^{2}=L^{2}$. Note also that the Lorenz model defines a certain linearly damped, driven symmetric top: to see this, set $a=0$ and $b=c=1$ in (5), and ignore all linear terms in (1b).

It is usually assumed that first integrals must be analytic or at least continuous [10] (however, see also Ref. [8] where nonanalytic functions as first integrals are also mentioned). This is an arbitrary restriction that is not always necessary in order to generate the transformation (4) over all finite times: every two-dimensional flow in phase space, including every driven-dissipative flow, is integrable via a conservation law, but that conservation law is typically singular [9]. "Nonintegrable" flows cannot occur in the phase plane. What can we say about "nonintegrability" in three or more dimensions?

In differential equations [10] and differential geometry [14] there is also an idea of local integrability: one can parallelize an arbitrary vector field $V$ about any point $x_{0}$ where the field $V(x)$ does not vanish. This means that we can "rectify" even chaotic and complex flows over a finite time, starting from any nonequilibrium point $x_{0}$. By analytic continuation [15], this local parallelization of the flow yields $n-1$ nontrival local conservation laws $y_{i}=G_{i}(x)=C_{i}$ that hold out to the first singularity of any one of the $n-1$ functions $G_{i}$. The singularities can only be branch points or phase singularities in the complex extension of phase space [16], so that a "nonintegrable" flow is 
always piecewise integrable: different sets of formulae of the form (4) hold for infinitely many consecutive finite time intervals $0 \leq t\left(x_{0}\right)<t\left(x_{1}\right)$, $t\left(x_{1}\right) \leq t<t\left(x_{2}\right), \ldots, t\left(x_{n-1}\right) \leq t<t\left(x_{n}\right), \ldots$, where the different points $x$ represent singularities of the first integrals. This explains Poincaré's observation that a dynamical system is generally neither integrable nor nonintegrable but is more or less integrable.

\section{DETERMINISTIC CHAOS AS SIMPLE DYNAMICS}

We have often read over the last twenty years that deterministic chaos can explain complex phenomena, but without having had an accompanying definition of "complex". In fact, chaotic dynamics can be understood from a topologic point of view as simple dynamics. This follows from a purely digital method called "symbolic dynamics".

Symbol sequences are abstractly equivalent to digit strings of numbers in some base of arithmetic. Here, computable numbers [17] are important because while "algorithmically random" numbers and sequences may "exist" in the mathematical continuum they would require infinite time and infinite precision for their definition, and therefore are irrelevant for both experiment and computation. If we use computable numbers as control parameters and initial conditions, then the chaotic dynamical systems typically studied in physics and chemistry are computable, e.g. via (2) combined with analytic continuation. The Lorenz model (1b) provides an example. Systems of chemical kinetic equations provide other examples. In contrast, the solution $x=x(0) \exp (t)$ of $\mathrm{d} x / \mathrm{d} t$ $=x$ is noncomputable unless both $x(0)$ and $t$ are computable numbers ("chaos" has nothing in particular to do with noncomputablility)

Using computable numbers, the local solution (2) of a dynamical system (1) that is digitized completely in some base of arithmetic defines an "artificial automaton", an abstract model of a computer $[9,17]$. The digitized initial condition constitutes the program for the automaton. In a chaotic dynamical system the part of the program that directs the trajectory into the distant future is encoded as the end-string $\varepsilon_{N+1} \ldots$ of digits in an initial condition $x_{0}=\varepsilon_{1} \varepsilon_{2} \ldots \varepsilon_{N} \ldots$ For example, the binary tent map, for every possible binary-encoded "computer program" $x_{0}$ $=\varepsilon_{1}(0) \varepsilon_{2}(0) \ldots \varepsilon_{N}(0) \ldots$, performs only a trivial computation: either it reads a bit in the program, or else flips the bit and reads it, then moves one bit to the right and repeats the operation $[9,17]$.

Unlike the binary tent map, which is exactly solvable in binary arithmetic, most dynamical systems do not admit a "natural" base of arithmetic. However, there is a systematic generalization of solution of the binary tent map via binary arithmetic that sometimes works: symbolic dynamics. The symbolic dynamics of a chaotic dynamical system can be defined, and solved digitally at least in principle, if the map has a "generating partition" [18]. For the binary tent map (6) the generating partition, in generation $n$, consists of the $2^{n}$ intervals $l^{(n)}=2^{-n}$ that are obtained by backward iteration of the unit interval by the map. Each interval in the generating partition can be labeled by an $n$-bit binary $(L, R)$ address called a symbol sequence. The symbol sequence tells us the itinerary of the map, for $n$ forward iterations, for any initial condition that is covered by the interval $l^{(n)}\left(\varepsilon_{1} \varepsilon_{2} \ldots \varepsilon_{n}\right)$ labeled by the $n$-bit address $\varepsilon_{1} \varepsilon_{2} \ldots \varepsilon_{n}$, where $\varepsilon_{i}=L$ or $R$ $[9,17]$.

Excepting pathological maps where the contraction rate in backward iteration is too slow, an infinite length symbol sequence corresponds uniquely to an infinitely precise initial condition. Given a symbol sequence, coarsegrained statistics for any number $N_{n}$ of bins in the generating partition $\left(N_{n}=2^{n}\right.$ for the binary tent map) can be obtained directly from the symbol sequence [17]. Those statistics depend strongly on initial conditions, and it is very easy to construct algorithms for initial conditions whose orbital statistics do not mimic uniform invariant densities (or 
"random" initial conditions). I have explained elsewhere why "random" or arbitrary initial conditions are a bad assumption for a dynamical system far from thermal equilibrium [16].

Because the binary tent map generates all possible infinite-length binary sequences (almost all of which are not computable via any possible algorithm), we can use that map to generate any histogram that can be constructed [17]. This result will be used below.

Liapunov exponents also depend on initial conditions. Chaotic dynamical systems like the Lorenz model or the logistic map generate an entire spectrum of Liapunov exponents $[9,17]$. We define a class of initial conditions to consist of all initial conditions that yield the same Liapunov exponent $\lambda$. Correspondingly, we can say that a class of symbol sequences defines a single Liapunov exponent. The Boltzmann entropy per iteration $s(\lambda)$ of all symbol sequences with the same Liapunov exponent $\lambda$ defines the fractal dimension $D(\lambda)=s(\lambda) / \lambda$ of that class of initial conditions $[9,17]$, so that a chaotic dynamical system generally generates spectra of both Liapunov exponents and fractal dimensions.

Both critical [19] and chaotic [17,18] dynamical systems may generate a natural partitioning of phase space, the generating partition, but not every nonintegrable dynamical system defines a generating partition. If a deterministic dynamical system has a generating partition then the symbolic dynamics can in principle be solved and the long-time behavior can be understood qualitatively, without the need to compute specific trajectories algorithmically from the algorithmic construction of a specific computable initial condition. In other words, a very high degree of "computational compressibility" holds even if the dynamical system is critical or chaotic.

Every chaotic dynamical system generates infinitely many different classes of statistical distributions for infinitely many different classes of initial conditions. The generating partition, if it exists, uniquely forms the support of every possible statistical distribution and also characterizes the particular dynamical system. For a system with a generating partition, topologic universality classes can be defined that permit one to study the simplest system in the universality class [18]. The infinity of statistical distributions is topologically invariant and therefore cannot be used to discern or characterize a particular dynamical system within a universality class [17]. The twodimensional Henon map belongs to the universality class of chaotic logistic maps of the unit interval peaking beneath unity [20], for example.

In such systems the long-time behavior can be understood qualitatively and statistically in advance, so that the future holds no surprises: the generating partition and symbol sequences can be used to describe the motion at long times, to within any desired degree of precision $l^{(n)}$, and multifractal scaling laws (via the $D(\lambda)$ spectrum) show how finer-grained pictures of trajectories are related to coarser-grained ones. Universality and scaling cannot describe complex dynamics, as we now discuss.

\section{COMPLEX DYNAMICS}

From the standpoint of computable functions and computable numbers we can and should think of a deterministic dynamical system as a computer with the initial condition as the program [9,17]. Thinking of dynamics from this point of view, it has been discovered that there is a far greater and far more interesting degree of complicated behavior in nonlinear dynamics than either criticality or deterministic chaos: systems of billiard balls combined with mirrors [21a,21b], and even two-dimensional maps [21c], can exhibit universal computational capacity via formal equivalence to a Turing machine. A system of nine first-order quasi-linear partial differential equations has been offered as a computationally universal system [22a,22b]. A quasi-linear firstorder partial differential equation in $n$ variables can be replaced by a linear one in $n+1$ variables. Maximum computational complexity is possible in 
systems of linear first-order partial differential equations. Such systems are nondiffusive but can describe damped-driven dynamics or wave propagation.

For a dynamical system with universal computational capability a classification into topologic universality classes is impossible [21c]. Given an algorithm for the computation of an initial condition to as many digits as computer time allows, nothing can be said in advance about the future either statistically or otherwise. One can at best compute the dynamics with controlled precision for that initial condition, iteration by iteration, to see what falls out. There is no computational compressibility that allows us to summarize the system's long-time behavior, either statistically or otherwise. In contrast with the case where topologic universality classes exist there is no organization of a hierarchy of periodic orbits, stable, marginally stable, or unstable, that allows us to understand the fine-grained behavior of an orbit from the coarse-grained behavior via scaling laws, or to look into the very distant future for special (so-called "random") initial conditions via symbolic dynamics. We do not know whether the Navier-Stokes equations or Newton's three-body problem fall into this category.

According to von Neumann [23] a system is complex when it is easier to produce than to describe mathematically. Under this qualitative definition the Henon map is not complex but a living cell is. In earlier attempts to model biologic evolution $[24,25]$ information was incorrectly identified as complexity. The stated idea was to find an algorithm that generates information, but this is too easy: both the square root algorithm and the logistic map $f(x)=4 x(1-x)$ generate information at the rate of one bit per iteration from rational binary initial conditions.

There is no physico-chemical model of the time-development of different degrees of complexity in nonlinear dynamics. No one knows if universal computational capability is necessary for biologic evolution, although DNA molecules in solution apparently are able to compute [26], but not error-free like an ideal Turing machine. Moore has speculated that computational universality should be possible in a certain kind of conservative three degree of freedom Newtonian potential flow [21c], but we do not know the minimum number of degrees of freedom necessary for universal computational capability in a driven-dissipative flow.

With a computationally universal (and therefore computable) dynamical system (1), given a specific computable initial condition $x_{0}$, both that initial condition and the dynamics can in principle be encoded as the digit string for another computable initial condition $y_{0}$. If the computable trajectory $y(t)=U(t) y_{0}$ could be digitally decoded, then we could learn the trajectory $x(t)=U(t) x_{0}$ for the first initial condition (selfreplication without copying errors). This maximum degree of computational complexity may be possible in low-dimensional nonintegrable conservative Newtonian dynamics. Some features of nonintegrable quantum systems with a chaotic classical limit (the helium atom, e.g.) have been studied using uncontrolled approximations based on the low-order unstable periodic orbits of a chaotic dynamical system [27], but we have no hint what might be the behavior of a low-dimensional quantum mechanical system with a computationally complex Newtonian limit.

\section{CAN NEW LAWS OF NATURE EMERGE FROM STUDIES OF COMPLICATED MOTIONS?}

The empirical discovery of mathematical laws of nature arose from the study of the simplest possible dynamical systems: Galilean trajectories of apples and Keplerian two-body orbits. Is there any reason to expect that simplicity can be shortcircuited in favor of complexity in the attempt to discover new mathematical laws of nature? Consider first an example from fluid dynamics where the attempt has been made to extract a simpler law of motion from a complicated time series. 
Fluid turbulence provides examples of complicated motions in both space and time in a Newtonian dynamical system of very high dimension. Ignoring harder problems by setting our sights low enough, we ask only the easiest question: is it possible in practice to extract from the data a simpler dynamical system that describes the transition to turbulence near criticality?

We have noted above that the binary tent map can generate all possible histograms that can be constructed simply by varying classes of initial conditions. Statistics that are generated by an unknown dynamical system are therefore inadequate to infer the dynamical law that generates the observed statistical behavior $[9,17]$. That is why, in any effort to derive a simplified dynamical system that describes either turbulence or the transition to turbulence, one cannot rely upon statistics. Instead, it is necessary to extract the generating partition of the dynamical system from the empirical data, if there is a generating partition.

Consider a low-dimensional dynamical system that has a generating partition. With infinite precision and infinite time, it would be possible in principle to pin down the map's universality class, and also the map, from a chaotic time series by the empirical extraction of the generating partition. With finite precision and finite time one must always resort to some guesswork after a few steps in the procedure. If one is to narrow down the practical choices to a few topologic universality classes of maps then the observational data must be extremely precise. Given the most accurate existing data on a fluid dynamical system near a critical point, the unique extraction of the universality class from an experimental time series has yet to be accomplished without physically significant ambiguity [28]. This demonstrates how difficult is the empirical problem that one faces in any attempt to extract an unknown law of motion from the analysis of critical or chaotic empirical data. For truly complex dynamical systems, the lack of a generating partition suggests that the extraction of laws of motion from empirical data is a hopeless task.

\section{IS SOCIO-ECONOMIC BEHAVIOR MATHEMATICALLY LAWFUL?}

Is it reasonable to expect that mathematical laws of socio-economic or other mathematical laws of human behavior exist? Is it possible abstractly to reduce some aspects of human behavior to a set of universal formulae, or even to a finite set of more or less invariant rules subject to simple or arbitrary initial conditions? By disregarding Galileo's historic and fruitful severing of the abstract study of inanimate motion from imprecise Aristotelian ideas of "motion" like youths alearning, acorns asprouting [2], and markets emerging, many mathematical economists have attempted to describe the irregularities of individual and collective human nature as if the price movements of a commodity, which are determined by human decisions and man-made political and economic rules, would define mathematical variables and abstract universal equations of motion analogous to ballistics and astronomy (deterministic models), or analogous to a drunken professor (stochastic models).

Mathematical economists often speak of the economy $[29,30]$, which is determined largely by human behavior and man-made rules, as if it could be studied mathematically as an abstract dynamical system like the weather. In the latter case the equations of motion are known but cannot be solved approximately over large space-time regions on a computer without the introduction of uncontrollable errors. However, for specified boundary and initial conditions the weather is determined by the mathematical equivalent of many brainless interacting bodies that cannot use intelligience to choose whether or not to obey the deterministic differential equations whose rigid mathematical rule they are condemned forever to follow.

Contrary to certain expectations [31] and extraordinary claims [32], there is presently no evidence to suggest that abstract dynamical systems theory can be used either to explain or understand socio-economic behavior. Billiard balls and gravitating bodies have no choice but to follow 
mathematical trajectories that are laid out deterministically, beyond the possibility of human convention, invention, or intervention, by Newton's laws of motion. The law of probability of a Brownian particle also evolves deterministically according to the diffusion equation beyond the possibility of human convention, invention, or intervention. In stark contrast, a brain that directs the movements of a body continually makes willful and arbitrary decisions at arbitrary times that cause it to deviate from and eventually contradict any mathematical trajectory (deterministic models) or evolving set of probabilities (stochastic models) assigned to it in advance. Two promenaders on a collision course can think and willfully alter their courses, whereas two billiard balls cannot. Given a hypothetical set of probabilities for a decision at one instant, there is no algorithm that tells us how to compute the probabilities correctly for later times, excepting at best the trivial case of curve-fitting at very short times, and then only if nothing changes significantly. Socio-economic statistics cannot be known in advance of their occurrence because, to begin with, there are no known socio-economic laws of motion that are correct.

Economists insist that they study open systems, whereas physics concentrates on closed systems, but this misses the point completely. We can describe and understand glaciers, tornadoes and hurricanes mathematically because the equations of thermo-hydrodynamics apply, in spite of the fact that the earth's atmosphere is an open dynamical system. We cannot understand the collapse of the Soviet Union or the financial crisis in Mexico on the basis of any known set of dynamics equations, in spite of the fact that the world economy forms a closed financial system.

Mathematical-lawlessness reigns supreme in the socio-economic fields, where nothing of any social or economic significance is left even approximately invariant by socio-economic evolution, including the "value" of the Mark. This is the reason that artificial law ("law") must be enforced by governments and central banks in the attempt to regulate economic behavior.

The division of observable phenomena into machine-like and not-machine-like behavior was made by Descartes [33]. In the Cartesian picture animals are supposed to behave more like machines, like robots that respond mechanically to stimuli. People, in contrast with robots, can reason and make decisions freely, or at least arbitrarily. Even the most illiterate or most stupid people can speak, can invent sentences creatively, and can behave unpredictably in other ways as well. The most intelligent dog, cat, or cow cannot invent intellectual complexity that is equivalent to a human language or a capitalist economy.

System theorists presume that an economy operates like the equivalent of an automaton that is too simple to simulate any kind of creative behavior, including the violation of politically enforced laws as occurred during the collapse of the former Soviet Union and the peasant rebellion in Chiapas. This is a strange assumption. Without human brains and human agreements based upon language, "laws" of economic behavior could not exist. Dogs, cows and even peasants generally do not invent money-economies. In contrast, the available geological and astronomical evidence suggests that Newton's laws and quantum mechanics held locally in our corner of the universe long before human languages emerged on earth.

Why should any part of nature behave mathematically, simulating a fixed automaton with a fixed program? Why can mathematics be used to describe the motions studied in physics, but not the "motions" (in Aristotle's sense) studied in economics, political science, psychology, and sociology?

\section{REDUCTIONISM, INVARIANCE PRINCIPLES, AND LAWS OF NATURE}

Reductionism is the arbitrary division of nature into laws of motion and initial conditions, plus "the environment". We must always be able to neglect "the environment" to zeroth order, because 
if nothing can be isolated then a law of motion can never be discovered. On the other hand, there are no laws of nature that can tell us the initial conditions. Laws of motion themselves obey laws called invariance principles, while initial conditions are completely lawless [34].

"It is not necessary to look deeper into the situation to realize that laws of nature could not exist without principles of invariance. This is explained in many texts of elementary physics even though only few of the readers of these texts have the maturity necessary to appreciate these explanations. If the correlations between events changed from day to day, and would be different for different points of space, it would be impossible to discover them. Thus the invariances of the laws of nature with respect to displacements in space and time are almost necessary prerequisites that it be possible to discover, or even catalogue, the correlations between events which are the laws of nature." See [34].

The experiments that Wigner had in mind are the parabolic trajectories of apples and blocks sliding down inclined planes, the two physical systems originally studied by Galileo in his empirical discovery of the local versions of Newton's first two laws of motion. Those discoveries would have been impossible in the absence of the geometric invariance principles of physics.

The observations that led to the discovery of the laws of inertia and gravity are precisely reproducible because absolute position and absolute time are irrelevant as initial conditions, which is the same as saying that space is homogeneous and isotropic (translational and rotational invariance) and that the flow of time is uniform. Socio-economic phenomena are not invariant: the statistics depend upon absolute position and absolute time, which is the same as admitting that socio-economic "motions" are not reducible to any known dynamical system.
If absolute time and absolute position were relevant initial conditions then identically prepared experiments would yield entirely different outcomes in different places and at different times. In this case physics would have remained Aristotelian and consequently would have evolved like economics, sociology, psychology, and political science: the study of a lot of special cases with no universal time-evolution laws, and consequently with no qualitative understanding whatsoever of the phenomena underlying the observed "motions" and their corresponding statistics. Arbitrary chaotic and complex modeling cannot shortcircuit this gap.

Mathematical laws that are determined by regularities of nature differ markedly from humancreated systems of merely conventional behavior. The latter consist of learned, agreed-on, and communally or politically enforced behavior, which can always be violated by willful or at least clever people. Even the usual idea of progress is a "motion" only in the Aristotelian sense.

Wigner considers that we cannot rule out that "holistic" laws of nature (beyond general relativity, for example) might exist, but if so then we have no way to discover them. Reductionism cannot explain everything mathematically, but reductionism is required in order to explain the phenomena that can be understood mathematically from the human perspective. Maybe an "oracle" would be required in order to discern the workings of a holistic law of motion.

\section{DARWINISM AND NEO-DARWINISM ${ }^{1}$}

"From a physicist's viewpoint, though, biology, history, and economics can be viewed as dynamical systems." See [32].

The Aristotelian dream of a holistic approach to physics, biology, economics, history, and other

\footnotetext{
${ }^{1}$ Kelly's book "Out of control" [35] contains many "paradigms" of postmodernist "holistic" thought.
} 
phenomena was revived by Bertalanffy in 1968 [36] under the heading of system theory. System theory proposes to use mathematics to describe the time evolution of "the whole", like a living organism or a money-economy, but generally in the absence of adequate information about the local correlations of the connected links that determine the behavior of the whole.

"Reductionism" is criticized by "holists" (see the introduction to Ref. [37] and also [38]; a physicist's attempt at holism is described in [39]). Some holists hope to be able to mathematize Darwinism $^{2}$ or neo-Darwinism (see discussions of "complex adaptable systems" [32]), but so far they have not been able use their postulated models to predict or explain anything that occurs in nature.

I call attempts to quantify the Aristotelian style of thought "reductionist holism", or "holistic reductionism" because any mathematization whatsoever is an attempt at reductionism. Quantification necessarily ignores all nonquantifiable qualities, and there are enough qualitative and quantitative considerations to ignore if we want to restrict our considerations to a definite mathematical model.

Some physicists tend to believe that physics, which is successful reductionism (often with several-to-high decimal accuracy in agreement between theory and reproducible observations), provides the basis for understanding everything in nature, but only in principle [40]. There is no effective way to "reduce" the study of DNA to the study of quarks but this is not a failure of reductionism: both quarks and DNA are accounted for by quantum mechanics at vastly different length scales. In order to adhere to the illusion that reductionism might also be able to account for social phenomena, in principle, physicists must leave out of consideration everything that has not been accounted for by physics, which includes most of the practical problems that ordinary people face in everyday life. When sociologists $[41,42]$ (who, unlike physicists, claim to interest themselves in the doings of ordinary people) try to follow suit but merely postulate or talk about dynamics "paradigms" in the absence of empirically established invariance principles, then they reduce their considerations of society to groundless mathematical models, to artificial simulations of life that have nothing to do with any important quality of life.

Mathematical simulations cannot adequately describe real societies and real economies although, through adequate politico-financial enforcement (a form of selection) we can be constrained to simulate an economist's simulation of society and economics (recall Marxism). A modern capitalist economy also represents a selection, one based upon material resources and human needs, desires, and illusions. The idealized free market system described by Adam Smith's "invisible hand" represents a vague notion of autonomy, or self-regulation, inspired in part by Calvinism and in part by Watt's flywheel governor, but is in no scientific sense a "natural" selection.

Darwin's ideas of "natural selection, fitness, and adaptation" may appear to make sense in both sports and the socio-economic context of daily life but they are not scientifically defined mathematical terms. That they remind us of the description of an organized market economy is not accidental: Darwin was strongly influenced during the cruise of the Beagle by his second reading of Malthus [43], who was both a protestant preacher and worldly philosopher. Terms like "selection" and "adaptation" are reminiscent of Adam Smith's vague "invisible hand" rather than of scientifically well-defined processes like the dissociation and recombination of DNA molecules described by quantum mechanics or chemical kinetics.

In an attempt to model the origin of life, chemical kinetic equations have been used to try

\footnotetext{
${ }^{2} \mathrm{Or}$, as one pundit put it, to Darwinize mathematics.
} 
either to discover or invent Darwinism at the molecular level [25] but the use of that terminology seems forced: a deterministic system of ordinary differential equations, whether chemical kinetic or not, can be described by the standard terminology of dynamical systems theory (stability, attractors, etc.). A stochastic system of chemical kinetic equations can be described by dynamics terminology combined with additional terms like "most probable distribution" and "fluctuations". There is less reason to believe that Darwin's socio-economic terminology applies at the macromolecular level than there was, before 1925, to believe that the language of the Bohr model correctly described the motions of electrons relative to nuclei in hydrogen and helium atoms.

There are two main sources of Darwin's vague notion of "natural selection". The social-Darwinist origin of the phrase is Malthus's socioeconomic doctrine, which derives from Calvinism [43] and can be traced through the late medieval revival of puritanism by Luther, Calvin, and Zwingli back to the neo-Platonist St. Augustine [6], who bequeathed to the west a religious notion of selection called "predestination". The second and only scientific motivation for Darwin's vague idea of "natural selection" came from plant and animal breeding, which he mis-labeled as "artificial selection". Plant and animal breeding constitute the only true case of selection because they proceed via manipulating certain initial conditions in order to try to achieve a desired result. Darwinists and neo-Darwinists, true to their nonGalilean heritage and worldview, are condemned to argue endlessly to try to find out what their terminology means.

The scientific foundation of organic evolution was established in Darwin's time by Mendel, who chose to become an Augustinian monk out of financial necessity [44] and was trained more in mathematics and physics than in biology. In contrast with Augustine, Luther and Calvin, Mendel was a lecturer in experimental physics, and approached the problem of heredity via iso- lation of cause and effect in the spirit of a physicist, or a good mechanic.

Darwin and his contemporaries, in contrast, accepted a holistic (or "integrated") picture of heredity that made the understanding of genetics impossible [45]. It was only after Mendel's reductionist discovery that some biologists began to dislodge themselves from the teleological notion of organic evolution as progress toward a goal predetermined by a selector (or read by an "oracle" capable of "infinite knowledge" of both future and past). By ignoring "the whole" in favor of the most important parts inferred by performing very simple, controlled experiments, Mendel found the key that divorced the study of heredity from unsystematic tinkering and socio-economic doctrine, and changed it into a precise mathematical science.

Contrary to Bak and Paczuski [32], we observe that human history is narrative. This includes the statistics of socio-economic phenomena, which constitute only one very small part of the entire narrative, a quantitative part. There is no reason to expect that the uncontrolled approximations of system theory modeling can tell us as much, quantitatively or qualitatively, about social or individual behavioral phenomena as we can learn from experience and by reading history and novels (see [46] for an uncontrolled approximation to the description of the consequences of the unrestricted drive toward economic efficiency). The reason why it is illusory to expect to discover objective laws of human history, including the history ("time-evolution") of socio-economic development, was explained prosaically in 1952:

"There can be no 'pure history'-history-initself, recorded from nobody's point of view, for nobody's sake. The most objective history conceivable is still a selection and an interpretation, necessarily governed by some special interests and based on some particular beliefs. It can be more nearly objective if those interests and beliefs are explicit, out in the open, where they can be freely examined and criticized. Historians can more 
nearly approach the detachment of the physicist when they realize that the historical 'reality' is symbolic, not physical, and that they are giving as well as finding meanings. The important meanings of history are not simply there, lined up, waiting to be discovered." See [47].

\section{ONE-DIMENSIONAL EXPECTATIONS}

"The nineteenth century, in western Europe and North America, saw the beginning of a process, today being completed by corporate capitalism, by which every tradition which has previously mediated between man and nature was broken." See [48].

A related viewpoint was developed earlier by the historian Spengler [49], who attempted to discover evidence for a grand scheme according to which human history evolves. Following the antiNewtonian Goethe, Spengler imagined human societies as "organisms" moving toward a "destiny". "Destiny" represents a vague idea of organic determinism that Goethe assumed to be in conflict with mechanistic time-evolution that proceeds via local cause and effect. "Destiny" was imagined to be impossible to describe via mathematical ideas, via Newtonian-style mechanism. In trying to make a distinction between global "destiny" and local cause and effect Spengler was not aware of the idea of attractors in dynamical systems theory, whereby time evolution mimics "destiny" but proceeds purely mechanically according local cause and effect. The Lie-Klein idea of invariance of geometry under coordinate transformations may have inspired Spengler's attempt to compare entirely different cultures, widely separated in time and space, as they evolved toward "destinies" that he identified as fully developed civilizations.

Spengler characterized western (European/ North American) civilization in the following way: the entire countryside is dominated by a few extremely overpopulated cities called mega- lopolises. Traditional cultures, derived from man's historic experience of wresting survival directly from nature, have been replaced by the abstract driving force of late civilization, the spirit of money-making. Spengler identified the transition from early Greek culture to late Roman civilization as an earlier example of the evolution from local tribal culture to money-driven civilization.

In our present civilization, in a single uncontrolled approximation, all traditions and ideas that interfere with "progress" defined as largescale and efficient economic development are rejected as unrealistic or irrelevant in the face of a one-dimensional quantitative position whose units may be dollars or marks. The dialogue paraphrased below can be found on page 16 of the book Complexity, Metaphors, Models, and Reality [37] about complex adaptable systems in biology, economics, and other fields. $\mathrm{A}, \mathrm{A}^{\prime}$, and $\mathrm{A}^{\prime \prime}$, who are paraphrased, are theoretical physicists.

A: Why try to define measures of complexity? A measure of complexity is just a number and that doesn't tell you anything about the system. Assume that there's a particular state that you want to create, a slightly better state of the economy, for example. Suppose that you want to know how complicated that problem is to solve on a computer, and that you're able to characterize complexity. One of the proposals of $\mathrm{A}^{\prime}$ for defining the complexity of a problem is 'what's the minimum amount of money you'd need in order to solve it?'

$\mathrm{A}^{\prime \prime}$ : The cost is proportional to computer time.

A: Then maybe the unit of complexity should be "money". If you're able to formalize the difficulty of solving the problem of making the economy slightly better, and you find out that you can measure its complexity in terms of dollars or yen, then that kind of measure would be extremely useful.

To the contrary, I expect instead that the complexity of a dynamical system, like fractal dimensions and Liapunov exponents, cannot be described by a single number. 


\section{Danksagung}

Dieser Aufsatz basiert teilweise auf meinem Eröffnungsvortrag beim Winter seminar März 1996 auf dem Zeinisjoch. Mein Dank gilt Professor Dr. Peter Plath, der mich zu diesem Vortrag eingeladen hat und auch $\mathrm{zu}$ dieser schriftlichen Form ermunterte, sowie Familie Lorenz für ihre liebenswürdige Bewirtung auf dem Zeinisjoch. Obgleich der größte Teil des Seminars auf Deutsch abgehalten wurde, entschied ich mich doch, meinen Vortrag auf Englisch zu halten. Mein Deutsch hätte doch nicht ausgereicht, komplizierten Gedankengänge präzise darzustellen wiederzugeben.

\section{References}

[1] R.S. Westfall (1980) Never at Rest, A Biography of Isaac Newton, ch. 1 (Cambridge Univ. Pr., Cambridge).

[2] J.L. Casti (1990) Searching for Certainty, What Scientists Can Know About the Future. (Wm. Morrow \& Co., New York).

[3] P.A. Samuelson (1976) Economics (McGraw-Hill, New York).

[4] R.P. Feynman (1986) Surely You're Joking Mr. Feynman (Bantom, New York) pp. 308-317.

[5] J.L. Casti (1992) Reality Rules: Picturing the World in Mathematics, in two volumes (Wiley, New York).

[6] A. Koestler (1959) The Sleepwalkers (Macmillan, New York).

[7] A. Wintner (1941) The Analytical Foundations of Celestial Mechanics (Princeton, Princeton) sect. pp. 194-202 \& pp. $227-240$.

[8] V.I. Arnol'd, V.V. Kozlov and A.I. Neishtadt (1993) Mathematical Aspects of Classical and Celestial Mechanics, in: Dynamical Systems III, Ed. V.I. Arnold (Springer-Verlag, Heidelberg).

[9] J.L. McCauley (Feb., 1997) Classical Mechanics: Flows, Transformations, Integrability, and Chaos (Cambridge Univ. Pr., Cambridge).

[10] V.I. Arnol'd (1981) Ordinary Differential Equations (M.I.T. Press, Cambridge, MA).

[11] H. Poincaré (1993) New Methods of Celestial Mechanics (AIP, Woodbury, NY).

[12] L.P. Eisenhart (1961) Continuous Groups of Transformations (Dover, New York).

[13] M. Tabor et al. (1991) in: What is Integrability?, Ed. V.E. Zakharov (Springer-Verlag, Berlin).

[14] P.J. Olver (1993) Applications of Lie Groups to Differential Equations (Springer-Verlag, New York) p. 30.

[15] J. Palmore (1995) private conversation.

[16] J.L. McCauley Nonintegrability, chaos and complexity, to appear in Physica A (1997).

[17] J.L. McCauley (1993) Chaos, Dynamics, and Fractals: An Algorithmic Approach to Deterministic Chaos (Cambridge Nonlinear Science Series 2, Cambridge).
[18] P. Cvitanovic, G. Gunaratne and I. Procaccia (1988) Phys. Rev. A 38 (1503).

[19] M.J. Feigenbaum (1988) J. Stat. Phys. 52, 527.

[20] G.H. Gunaratne (1990) Universality beyond the onset of chaos, in: Chaos: Soviet and American Perspectives on Nonlinear Science, Ed. D. Campbell (AIP, New York).

[21] (a) E. Fredkin and T. Toffoli (1982) Int. T. Theor. Phys. 21, 219; (b) C. Bennett (1982) Int. J. Theor. Phys. 21, 905; (c) C. Moore (1990) Phys. Rev. Lett. 64, 2354.

[22] (a) S. Omohundro (1984) Physica 10D, 128; (b) C. Bennett (1986) Found. Phys. 16, 585.

[23] J. von Neumann (1966) Theory of Self-Reproducing Automata (Univ. of IL., Urbana) pp. 47, 48.

[24] M. Eigen and R. Winkler-Oswatitsch (1987) Stufen zum Leben (R. Piper, München).

[25] B.-O. Küppers (1985) The Molecular Theory of Evolution (Springer, Heidelberg).

[26] R.J. Lipton (1995) Science 268, 542.

[27] H. Friedrich (Apr. 1992) Physics World, 32-36.

[28] A. Chhabra, R.V. Jensen and K.R. Sreenivasan (1989) Phys. Rev. A 40, 4593.

[29] J.L. Casti and A. Karlqvist (1991) Beyond Belief: Randomness, Prediction, and Explanation in Science (CRC Press, Boca Raton).

[30] P.W. Anderson, K.J. Arrow and D. Pines, Eds. (1988) The Economy as an Evolving Complex System (AddisonWesley, Redwood City).

[31] D. Ruelle (July, 1994) Physics Today, 24-30.

[32] P. Bak and M. Paczuski (1995) Complexity, Contingency, and Criticality, subm. to Proc. Nat. Acad. Sci.

[33] N. Chomsky (1968) Language and Mind (Harcourt Brace, Janovich, New York).

[34] E.P. Wigner (1967) Symmetries and Reflections (Univ. Indiana Pr., Bloomington).

[35] K. Kelly (1994) Out of Control (Addison-Wesley, Reading) ch. 22 .

[36] L. von Bertalanffy (1968) General Systems Theory (G. Braziller, New York)

[37] G.A. Cowan, D. Pines and D. Meltzer, Eds. (1994) Complexity, Metaphors, Models, and Reality (Addison-Wesley, Reading).

[38] H. Morowitz (1995) Complexity 1, 4.

[39] P. Bak (1994) Self-Organized Criticality: A Holistic View of Nature in ref.

[40] S. Weinberg (1995) Reductionism Redux, New York Review of Books, pp. 39-42.

[41] R.L. Ackoff (1974) Redesigning the Future (WileyInterscience, New York).

[42] R. Collins (1988) Theoretical Sociology (Harcourt Brace, Janovich, San Diego).

[43] R.M. Young (1985) Darwin's Metaphor (Cambridge Univ. Pr., Cambridge)

[44] R. Olby (1985) Origins of Mendelism, 2nd Edn. (Univ. of Chicago Pr., Chicago).

[45] P.J. Bowler (1989) The Mendelian Revolution (Johns Hopkins, Baltimore)

[46] K. Vonnegut (1952) Player Piano (Avon Books, New York).

[47] H.J. Muller (1952) The Uses of the Past (Oxford Univ. Pr., New York).

[48] J. Berger (1991) About Looking (Vintage Pr., New York).

[49] O. Spengler (1922) Untergang des Abendlandes (C. H. Bech'sche Verlagsbuchhandlung, München). 


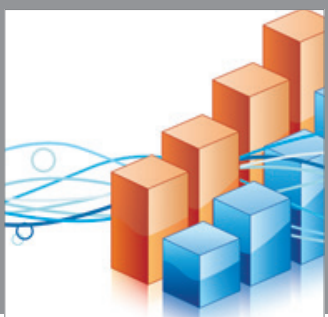

Advances in

Operations Research

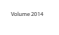

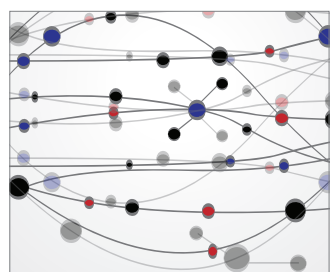

\section{The Scientific} World Journal
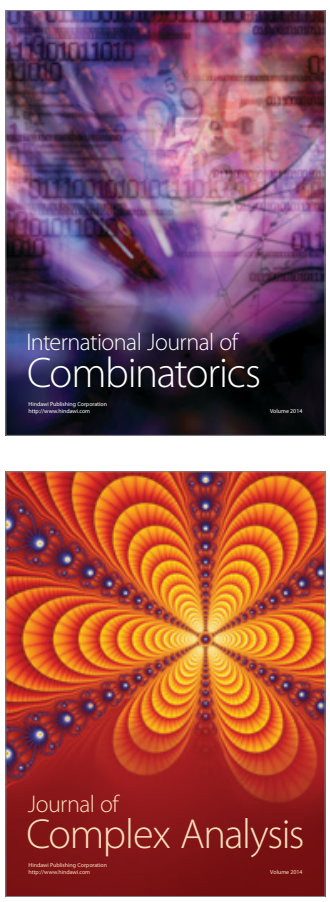

International Journal of

Mathematics and

Mathematical

Sciences
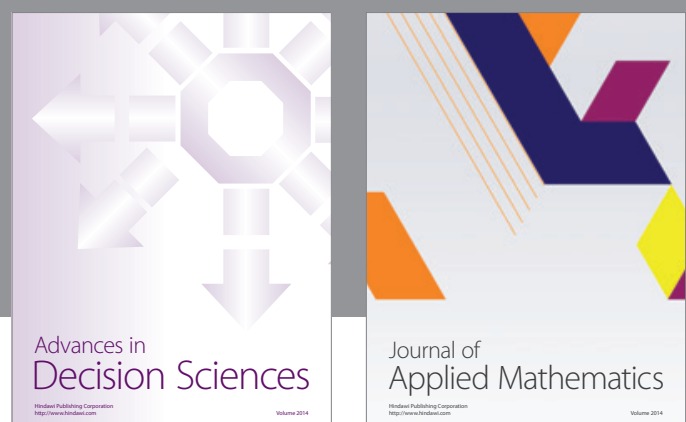

Journal of

Applied Mathematics
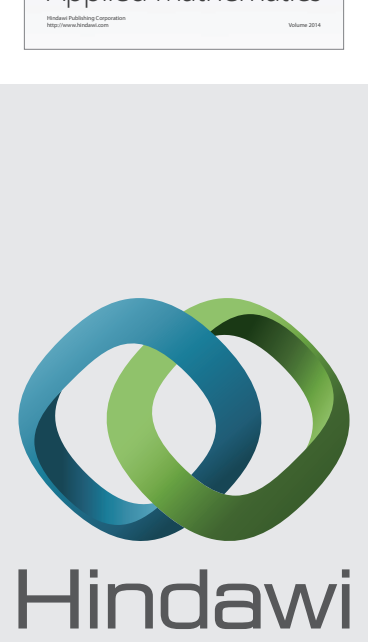

Submit your manuscripts at http://www.hindawi.com
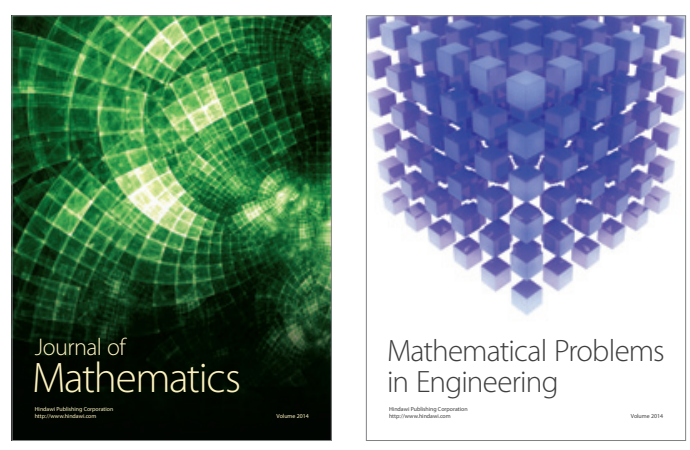

Mathematical Problems in Engineering
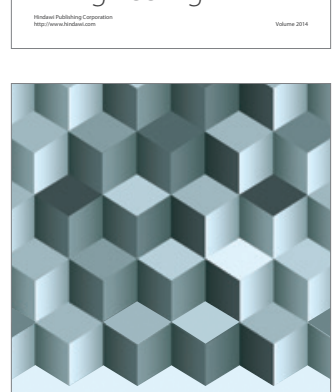

Journal of

Function Spaces
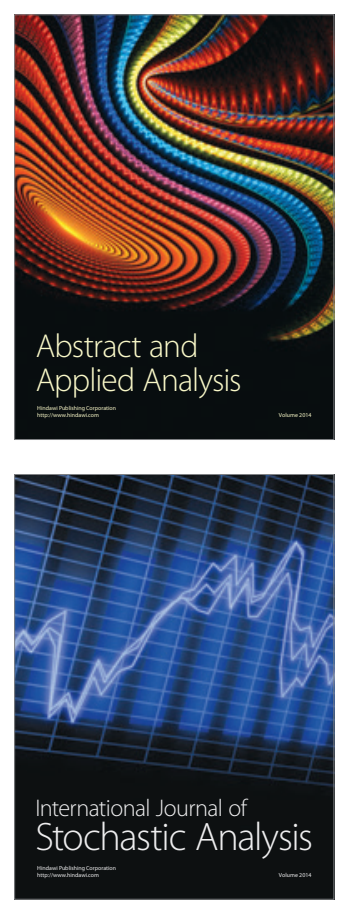

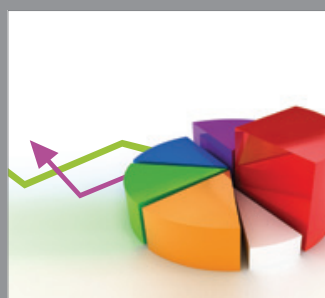

ournal of

Probability and Statistics

Promensencen
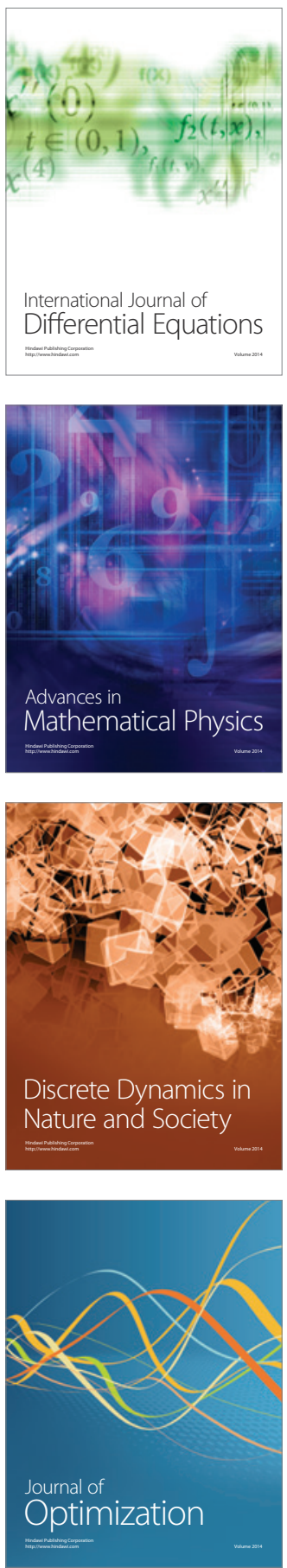